\section{Explaining the exceptions}

\section{By Chris Cain, Senior Writer}

A Boston team has identified activating mutations in mammalian target of rapamycin that explain a surprising 14-month complete response seen in 1 patient with bladder cancer receiving Afinitor and Votrient in a Phase I trial. ${ }^{1}$ The findings are the latest in a string of studies characterizing 'exceptional responders'. This year the National Cancer Institute plans to launch an initiative to sequence 100 such patients to help identify additional mutations that could prospectively predict drug response.

According to the NCI, up to $10 \%$ of patients in Phase II clinical trials of new drugs respond well to therapeutics that ultimately are not approved for the indication. Although a precise molecular explanation for the response in these patients often is a mystery, a series of high-profile publications have shown how next-generation sequencing can provide answers.

In one example from researchers at the Memorial Sloan-Kettering Cancer Center, a patient with bladder cancer with a two-year durable response to Afinitor everolimus was shown to have a mutation in tuberous sclerosis complex tumor suppressor 1 (TSC1), a regulator of mammalian target of rapamycin (mTOR; FRAP; RAFT1) activation. Results were published in Science. ${ }^{2}$

The patient was a participant in an investigator-initiated Phase II trial that missed its primary endpoint.

Afinitor, an oral mTOR inhibitor from Novartis AG, is marketed to treat brain cancer, breast cancer and neuroendocrine tumors. Novartis is not sponsoring trials of Afinitor to treat bladder cancer.

In another example, the tyrosine kinase inhibitor Sprycel dasatinib led to a multiyear durable response in a patient with non-small cell lung cancer (NSCLC) in a Phase II study, whereas the other 33 patients in the trial did not respond. Genomic and functional analyses showed that the patient's tumor carried an inactivating mutation in $B R A F$ that explained the sensitivity. Results were published in Science Translational Medicine. ${ }^{3}$

Bristol-Myers Squibb Co. and Otsuka Pharmaceutical Co. Ltd. market Sprycel to treat acute lymphoblastic leukemia (ALL) and chronic myelogenous leukemia (CML). Bristol-Myers Squibb is running a Phase II study of Sprycel in subsets of genetically defined patients with NSCLC, including those with inactivating $B R A F$ mutations.
The latest example came this month from a DanaFarber Cancer Institute investigator-initiated Phase I study of Afinitor plus Votrient pazopanib, a VEGF inhibitor that GlaxoSmithKline plc markets to treat renal cell carcinoma (RCC) and advanced soft tissue sarcomas.

Jonathan Rosenberg, who was the principal investigator on the trial, told SciBX, "Phase I studies don't generally get complete responses, so when a patient with heavily pretreated metastatic bladder cancer had complete remission assessed by imaging at about 14 months, we took note."

Rosenberg ran the study when he was clinical director of the Genitourinary Oncology Center and director of the Bladder Cancer Center at Dana-Farber and Brigham and Women's Hospital. He now is section head of nonprostate genitourinary cancers at MSKCC.

To identify mutations that could explain the complete response, Rosenberg did whole-exome sequencing of the patient's pretreatment tumor sample and compared it with germline DNA. He identified two tumor-specific, concurrent, activating mutations in mTOR that had not been previously seen in human cancers.

In cell culture, the mTOR inhibitor rapamycin reduced the activity of these mutant forms of the protein, whereas Votrient had no effect. Thus, the response was likely due to Afinitor sensitivity.

Results were published in Cancer Discovery. The work was done in collaboration with Levi Garraway, who is an associate professor of medicine at Harvard Medical School, an associate professor of medicine at Dana-Farber and a senior associate member at the Broad Institute of MIT and Harvard.

A separate, concurrent publication in the journal described 33 mTOR activating mutations that were identified through analysis of publicly available cancer genome datasets, suggesting that additional mutations may also confer sensitivity to mTOR inhibition. ${ }^{4}$

\section{Expanding the search}

The NCI and other institutions are now dedicating resources to sequence additional patients who have had extraordinary responses.

Barbara Conley, associate director of the Cancer Diagnosis Program at the NCI, said that a review of the institute's sponsored early clinical trials of targeted therapies from the last 10 years found about 100 patients with an extraordinary or exceptional response to therapy.

NCI defines an exceptional response as a complete response to 
treatment or a partial response lasting at least six months for a drug that did not go on to receive FDA approval in that indication because of insufficient activity.

"We took a look at those trials at NCI the last 10 years or so, excluded the straight chemotherapy drugs and agents which went on to be successfully approved in the tested indication, and we found somewhere between $3 \%-10 \%$ of patients on these trials were exceptional responders," said Conley.

This summer, the NCI plans to start the pilot Exceptional Responders Initiative to sequence about 100 exceptional responders, including those solicited from Phase I and II studies of targeted therapies as well as patients who had an exceptional response to standard systemic treatments. The NCI will reimburse sites for the cost of sending pretreatment tumor samples and clinical data. Specimens will be sent to a biospecimen core facility at the Nationwide Children's Hospital, where specimens will be prepared and nucleic acids isolated. A sequencing and characterization center will conduct whole-exome and other sequencing on these samples.

"We hope to get samples from many different clinical sites, and we are trying to make it as simple as possible to submit them through the cancer trials support unit. But it does take time to get the samples, prepare them and sequence them. Patients, if living, will have given appropriate consent for use of their tissue in this way or we will ask for patient consent," said Conley.

She noted that in many cases the ability to obtain pretreatment tissue samples will be a limiting factor. She expects about one-quarter to onethird of trials will have tissue available for sequencing.

Data will be deposited into a publicly accessible database, but individual patients will not be identified.

Rosenberg said that he is also collaborating with Garraway on additional work characterizing bladder cancer genomes. The duo is participating in a multicenter effort to sequence patients to understand response to chemotherapy, which is funded by a grant from the Starr Cancer Consortium.

Conley said that the NCI initiative is interested in targeted systemic therapies but will include the study of patients with extraordinary chemotherapy responses as well.

Rosenberg added that although retrospective studies such as these are highly valuable to identify new drug sensitivities, cancer centers such as MSKCC are beginning to sequence patients' tumors up front to prospectively identify potential sensitivities.
The NCI is gearing up to launch the NCI-MATCH (Molecular Analysis for Therapy Choice) clinical trial in November. The study will consist of about 20 arms and will enroll molecularly characterized patients with metastatic disease or locally advanced disease that has been refractory to treatment.

The characterization will include targeted sequencing of variants of about 200 genes, amplifications and targeted translocations, as well as additional immunohistochemistry and fluorescence in situ hybridization (FISH) tests. The NCI is in discussion with pharma and biotech companies about which agents to test.

The initiative will be led by the cooperative group ECOG-ACRIN and National Clinical Trials Network groups and will include participation from over 3,000 clinical sites.

Cain, C. SciBX 7(12); doi:10.1038/scibx.2014.332

Published online March 27, 2014

\section{REFERENCES}

1. Wagle, N. et al. Cancer Discov.; published online March 13, 2013; doi:10.1158/2159-8290.CD-13-0353

Contact: Jonathan E. Rosenberg, Memorial Sloan-Kettering Cancer Center, New York, N.Y.

e-mail: rosenbj1@mskcc.org

Contact: Levi A. Garraway, Dana-Farber Cancer Institute, Boston, Mass.

e-mail: levi_garraway@dfci.harvard.edu

2. Sen, B. et al. Sci. Transl. Med. 4, 136ra70 (2012)

3. Iyer, G. et al. Science 338, 221 (2012)

4. Grabiner, B.C. et al. Cancer Discov.; published online March 14, 2013; doi:10.1158/2159-8290.CD-13-0929 Contact: David M. Sabatini, Whitehead Institute for Biomedical Research, Cambridge, Mass.

e-mail: sabatini@wi.mit.edu

\section{COMPANIES AND INSTITUTIONS MENTIONED}

Brigham and Women's Hospital, Boston, Mass.

Bristol-Myers Squibb Co. (NYSE:BMY), New York, N.Y.

Broad Institute of MIT and Harvard, Cambridge, Mass.

Dana-Farber Cancer Institute, Boston, Mass.

GlaxoSmithKline plc (LSE:GSK; NYSE:GSK), London, U.K.

Harvard Medical School, Boston, Mass.

Memorial Sloan-Kettering Cancer Center, New York, N.Y.

National Cancer Institute, Bethesda, Md.

Nationwide Children's Hospital, Columbus, Ohio

Novartis AG (NYSE:NVS; SIX:NOVN), Basel, Switzerland

Otsuka Pharmaceutical Co. Ltd., Tokyo, Japan

Starr Cancer Consortium, New York, N.Y. 